\title{
Diuretics and vitamin B1: are diuretics a risk factor for thiamin malnutrition?
}

\author{
Suter, Paolo M ; Vetter, Wilhelm
}

\begin{abstract}
Despite modern pharmacologic agents in the therapy of heart failure, the prevalence of heart failure is increasing worldwide. In the vitamin B1 deficiency disease beriberi, cardiac symptoms may represent the central feature. Two new studies confirmed that all diuretics lead to increased uri- nary thiamin excretion depending on the urinary flow rate. In a subject at risk, such as an elderly patient, chronic diuretic treatment may lead to a subclinical thiamin deficiency. Whether subclinical thiamin nutriture is a modulator of the prevalence andlor severity of heart failure is not known; however, it seems to be plausible from the metabolic point of view
\end{abstract}

DOI: https://doi.org/10.1111/j.1753-4887.2000.tb01827.x

Posted at the Zurich Open Repository and Archive, University of Zurich ZORA URL: https://doi.org/10.5167/uzh-153978

Journal Article

Published Version

Originally published at:

Suter, Paolo M; Vetter, Wilhelm (2009). Diuretics and vitamin B1: are diuretics a risk factor for thiamin malnutrition? Nutrition Reviews, 58(10):319-323.

DOI: https://doi.org/10.1111/j.1753-4887.2000.tb01827.x 


\section{Diuretics and Vitamin $B_{1}$ : Are Diuretics a Risk Factor for Thiamin Malnutrition?}

\begin{abstract}
Despite modern pharmacologic agents in the therapy of heart failure, the prevalence of heart failure is increasing worldwide. In the vitamin $B_{\text {, }}$ deficiency disease beriberi, cardiac symptoms may represent the central feature. Two new studies confirmed that all diuretics lead to increased urinary thiamin excretion depending on the urinary flow rate. In a subject at risk, such as an elderly patient, chronic diuretic treatment may lead to a subclinical thiamin deficiency. Whether subclinical thiamin nutriture is a modulator of the prevalence and/or severity of heart failure is not known; however, it seems to be plausible from the metabolic point of view.
\end{abstract}

Diuretics represent the pharmacologic comerstone in the therapy of hypertension and congestive heart failure. ${ }^{1}$ The therapeutic effect of diuretics is mainly mediate by increased urinary salt and volume excretion. ${ }^{2}$ The side effects of diuretics are well known, and one of the most important side effects of diuretics is hypokalemia owing to the increased urinary loss of potassium. ${ }^{2}$ All clinicians and many patients are aware of this side effect because hypokalemia may result in dangerous cardiac arrhythmias. Accordingly, the biochemical monitoring of plasma potassium levels represents a routine procedure in patients on diuretics. Nevertheless, it is less well known that diuretics also increase the excretion of other electrolytes, minerals, and water-soluble vitamins. ${ }^{3-11}$

The effect of diuretics on the excretion of watersoluble vitamins has been discussed repeatedly during the last 30 years; however, many clinicians and cardiologists usually ignore the importance of these findings. The effect of diuretics on urinary thiamin excretion was recently addressed in two publications. One can hope that these studies will lead to a revival of interest in this important aspect of vitamin nutriture and a clarification of the controversy.

Rieck et al. ${ }^{12}$ studied the effect of single intravenous $1 \mathrm{mg}, 3 \mathrm{mg}$, and $10 \mathrm{mg}$ doses of furosemide compared with normal saline infusions on urinary thiamin excretion in six healthy volunteers. The different furosemide doses and the saline infusions resulted in a dose-dependent increase in urine flow and sodium excretion, which was accompanied by an increase in the urinary thiamin excretion rate. The urinary thiamin excretion rate doubled during an initial observation period of 6 hours from a baseline thiamin

This review was prepared by Paolo M. Suter, M.D., M.S., and Wilhelm Vetter, M.D., Medical Policlinic, University Hospital, 8091 Zürich, Switzerland. excretion rate of $6.4 \pm 5.1 \mathrm{nmol} /$ hour to $11.6 \pm 8.2 \mathrm{nmol} /$ hour $(p<0.01)$. Thiamin excretion then returned to baseline levels during the following 18 hours. In these subjects, a significant correlation between the urinary thiamin excretion rate and the urine flow rate was observed $(r=0.54, p$ $<0.001){ }^{12}$

The second publication, from the same research group, tried to evaluate the mechanisms of furosemide-induced urinary thiamin excretion. ${ }^{13}$ In this study, Lubetsky et al. ${ }^{13}$ measured the urinary thiamin excretion in rats in response to increasing doses of furosemide, acetazolamide, chlorothiazide, amiloride, mannitol, and a saline infusion. In this rat study ${ }^{13}$ the thiamin excretion increased independently from the type of diuretic given and increased to a maximal value of $7.44-9.34 \mathrm{pmol} /$ minute $(p=0.003)$. There was no significant difference between the miscellaneous diuretics and the saline infusion. The thiamin excretion in this model correlated more or less linearly with the urinary volume, and the urinary flow rate explained $78 \%$ of the variability in thiamin excretion rates.

The classic vitamin $B_{1}$ deficiency disease is beriberi. ${ }^{14,15}$ In most Western countries the clinical entity of beriberi is only of historical importance, although at closer examination beriberi disease can be found in specific clinical settings, as reported in several articles and case reports. ${ }^{16-19}$ Beriberi disease has two major clinical manifestations: dry beriberi, prominently featuring encephalopathy and peripheral neuropathy, and wet beriberi, in which high output cardiac failure with peripheral edema represents the main clinical feature. ${ }^{20}$ Thiamin plays an important role in the function of the cardiac muscle and the maintenance of adequate heart function..$^{21-23}$ Theoretically, any condition leading to a suboptimal nutritional status of this vitamin may lead, in the long run, to impaired cardiac function.

The well-known deficiency diseases of thiamin are the Wernicke-Korsakoff syndrome and lactic acidosis..$^{24,25}$ Both conditions are well known to most clinicians, which may be one of the reasons that the classical beriberi heart disease has been forgotten during the last few decades.

There is no doubt that diuretics do increase the excretion of thiamin; nevertheless, there is controversy about the pathophysiologic and clinical importance of the increased urinary losses of thiamin. All studies reported the acute effects of diuretics on thiamin excretion. It is not known, however, whether chronic diuretic ingestion leads to renal adaptation with reduced urinary thiamin excretion.

By comparison with other vitamins, such as the lipidsoluble vitamins, thiamin body stores are rather small and whole-body thiamin stores are only approximately $30 \mathrm{mg}^{26}$ 
In addition, vitamin $B_{1}$ metabolism has a high turnover rate with a half-life of 10-18 days. These characteristics of the vitamin imply that for the maintenance of optimal nutriture a constant dietary supply of this vitamin must be assured. The constant dietary thiamin supply is easily achieved in healthy subjects eating according to the present guidelines. ${ }^{26}$ Certain subgroups in the population, however, are at high risk for the development of vitamin $\mathrm{B}_{1}$ deficiency; these populations include chronic alcohol consumers, the elderly, ${ }^{27,28}$ and probably elderly diuretic users.

In the present context several questions need to be addressed: What is the prevalence of thiamin deficiency? Is the effect of medical and pathophysiologic importance? What is the mechanism of the diuretic-induced increase in thiamin excretion? Can the diuretic effects be counteracted? Should diuretic consumers be supplemented with vitamin $\mathrm{B}_{1}$ ?

As mentioned above, any condition leading to a disruption of the continuous intake and/or loss of thiamin will favor the development of a deficiency state. Up to $80 \%$ of heavy alcohol consumers have been found to be thiamin deficient. ${ }^{27,29}$ Aging is associated with different alterations in thiamin metabolism, such as low intake and decreased capacity to activate the vitamin. ${ }^{30} \mathrm{Up}$ to $50 \%$ of elderly persons have been found to be deficient in thiamin. ${ }^{30}$ Chronic diseases, institutionalization, and hospitalization increase the risk for thiamin deficiency. Compared with other nutrients, thiamin respresents a nutrient with a very high potential for deficiency.

The exact mechanism of the increased excretion of thiamin by diuretics is not known. The studies by Rieck et al. ${ }^{12}$ and Lubetsky et al. ${ }^{13}$ suggest that the mechanism is nonspecific and urinary flow dependent. Lubetsky et al. ${ }^{13}$ compared the effect of different diuretics (furosemide, acetazolamide, chlorothiazide, amiloride) acting at different anatomic and functional levels of the renal tubules (loop of Henle, proximal tubule, distal convoluted tubule, collecting duct). In addition, they compared the effect of these diuretics with mannitol and simple extracellular volume expansion by the administration of saline, the latter two eliciting an effect on the whole nephron. Any increase in diuresis induced an increase in thiamin excretion independently from the applied strategy to increase urinary flow. ${ }^{13}$ This suggests that the phenomenon is only related to the increased urinary flow and not any specific effect on thiamin secretion or resorption. They report that urinary flow rates accounted for $78 \%$ of the variability in the thiamin excretion rates upon the different interven ${ }^{+}$ons. These rather strong data from the carefully performed studies by Lubetsky et al. ${ }^{13}$ and Rieck et al. ${ }^{12}$ are very supportive of the thiamin-loosing effect as a phenomenon of all diuretics. Nevertheless, in earlier studies potassium-sparing diuretics have not been found to increase urinary thiamin excretion. ${ }^{8,31}$ This discrepancy can be explained by methodologic issues regarding the design of the study and the animal model used, as well as the diuretic type and dosage applied. The suggested urinary flow dependency of the diuretic-induced thiamin loss also receives indirect support from evidence of thiamin deficiency in hemodialysis patients. ${ }^{32}$

Thiamin excretion in humans depends mainly on the blood thiamin concentration. Under normal conditions, i.e., in the absence of kidney disease and/or diuretic intake, at low blood levels hardly any thiamin is excreted because tubular reabsorption of this vitamin is enhanced in this clinical condition. ${ }^{33}$ With increasing intakes and blood levels, thiamin excretion rises sharply. According to the present evidence, diuretics seem to disrupt these regulatory mechanisms, thus increasing the risk for deficiency. The increased diuretic associated risk for vitamin $B_{1}$ deficiency underlines the importance of monitoring thiamin status when prescribing diuretics to subjects at risk, i.e., elderly persons.

The regulation of urinary excretion of thiamin is not well understood. Nevertheless, it is evident that the diuretic-induced increase in thiamin excretion in subjects with high plasma thiamin levels is probably of no clinical or pathophysiologic importance as long as high plasma levels can be maintained. In elderly subjects, higher plasma thiamin levels are not always easily achieved, thus predisposing them to a higher risk for thiamin deficiency when taking diuretics.

Several smaller studies reported impaired thiamin nutriture in patients with congestive heart failure and concomitant diuretic therapy. ${ }^{4,34}$ Some studies could not show a thiamin deficiency in patients with similar diagnoses. ${ }^{10,35,36}$ These inconsistent results are not surprising and probably can be explained by the metabolic characteristics of thiamin as well as by the potential differences in thiamin intake as a function of different eating habits (e.g., high-carbohydrate versus low-carbohydrate diets) and food preparation habits (e.g., long cooking and storing), and also by the different levels of physical activity of studied populations. ${ }^{3}$ In addition, most of the normal plasma values for vitamins-including vitamin $\mathrm{B}_{1}$ - are based on the concept of prevention of the classic deficiency diseases and not necessarily on functional parameters (such as heart function).

It is conceivable that plasma levels in the upper range of normal, or even supranormal, in patients with heart failure may lead to favorable pharmacologic effects at the level of the heart. Miscellaneous pharmacologic effects for vitamins have been reported and it is not far-fetched to suggest that normal plasma thiamin levels in heart failure patients may not be adequate for optimal organ function of the diseased heart. This concept is also supported by a small intervention trial reporting an improvement of left ventricular function in patients with congestive heart failure on diuretic therapy following short-term supplemen- 
tation with thiamin..$^{34}$ In patients with normal biochemical vitamin $B_{1}$ status, the parenteral short-term supplementation of $200 \mathrm{mg} /$ day for 1 week followed by a 6-week oral supplementation regimen with the same dosage led to significant improvement (by $22 \%$ ) of the left ventricular ejection fraction. The improvement of left ventricular function in this study ${ }^{34}$ was apparent after the first week of therapy. Although the response pattern in this study was very heterogeneous, the effect of the supplementation reached the level of significance.

The importance of functional testing in the assessment of a suboptimal nutritional status is very important. This is also reflected in the results of a recent study reporting increased homocysteine levels in hypertensive patients on long-term diuretic therapy. ${ }^{37}$ The increased homocysteine levels represent a marker for a functional impairment of vitamin-dependent metabolic clearing pathways of homocysteine. In the study by Morrow et al. ${ }^{37}$ diuretic use was associated with decreased plasma folate levels, although the plasma folate levels remained within normal limits. In view of the normal folate plasma jevels, no folate supplementation would be warranted. Nevertheless, the increased homocysteine levels represent a marker for a deficiency and a rather clear indication for supplementation. In a recent prospective study evaluating the change in nutritional status of patients during hospitalization in a medical clinic, a relationship between the cumulative diuretic dosage given during the hospitalization and the decline in vitamin $B_{1}$ nutriture was reported. ${ }^{38}$

Certain patients and population groups can cot obtain all essential nutrients from the diet alone, which is often also the case for vitamin $B_{1}$. The major food sources of vitamin $B_{1}$ are wheat germ, whole grain products, yeast, nuts, and meat products. Approximately $40 \%$ of dietary thiamin is ingested in the form of cereals and cereal products; it must be remembered, however, that the usual food processing techniques may considerably reduce the food content of this vitamin. ${ }^{39}$ Most chronic diuretic users are elderly persons, and it is not easy to recommend to this population group the ingestion of whole grain products, owing to potential digestion problems. In addition, thiamin is very heat labile, and depending on the cooking techniques up to $80 \%$ of the vitamin can be destroyed. ${ }^{39}$ Food services for elderly persons depend on rather long food preparation procedures (including cooking, storage, and transport while keeping the food hot), which lead to a considerable loss of this very heat-sensitive vitamin. A diet high in carbohydrates (mainly in the form of simple sugars) is associated with an increased risk of thiamin deficiency. ${ }^{6,16}$ Accordingly, it seems prudent to support the concept of pharmacologic improvement of vitamin $B_{1}$ nutriture in elderly patients on chronic diuretic therapy. A safe and ideal vitamin $B_{1}$ dosage for elderly patients on chronic diuretic therapy should be higher than the present dietary recommendations; a dosage of 20-50 mg/day seems reasonable.

Alcohol abuse represents the single most important risk factor for thiamin deficiency in Western countries. Owing to the high impact of alcohol on thiamin nutriture, fortification of alcoholic beverages has been suggested. ${ }^{40}$ It can therefore be hypothesized that a combined formulation of a diuretic with thiamin may bear some therapeutic advantage in certain clinical settings such as heart failure in elderly persons.

Despite the high potential for cardiac function improvement upon thiamin supplementation in elderly patients with cardiac failure, there may be insufficient clinical improvement of cardiac function in selected patients. There may be several causes for this unresponsiveness, such as disease progression, noncompliance with pharmacologic therapy, and magnesium deficiency. Chronic diuretic use may lead to an increased urinary loss of magnesium and subclinical magnesium deficiency. ${ }^{41-43}$ Magnesium plays a central role in heart muscle function ${ }^{44,45}$ and in the activation of thiamin to thiamin pyrophosphate, the active form of the vitamin. ${ }^{46}$ The prevalence of magnesium deficiency in chronic diuretic users may be rather high, predisposing them to an insufficient metabolic activation of vitamin $B_{1}$. Accordingly, to obtain an optimal clinical response to vitamin $B_{1}$ supplements, adequate magnesium status has to be maintained. The role of magnesium in thiamin metabolism is also nicely supported in a case report describing the occurrence of Wernicke's encephalopathy induced by magnesium deficiency. ${ }^{47}$ In the present context, it must be remembered that potassiumsparing diuretics do have magnesium-conserving properties. ${ }^{43}$ Aging is also regarded as a risk factor for magnesium deficiency, ${ }^{48}$ thus enhancing the risk for an impairment of thiamin status.

Besides interacting at the level of the kidney, some cardiac drugs may interfere with thiamin metabolism at the level of the cardiac cells. It has been reported that furosemide and digoxin inhibit thiamin uptake by cardiac cells in culture, ${ }^{49}$ which would represent another potentially unfavorable effect of diuretics on thiamin nutriture and heart function. The clinical importance of the effect of diuretics on thiamin uptake by cardiac cells is not known.

Pharmacoepidemiologic data underline the potential importance of diuretics in the present context. More than $50 \%$ of elderly subjects ( $>65$ years) received diuretics in a recent survey from the Netherlands. ${ }^{1}$ The prescription rates of loop diuretics increased from $15 \%$ in patients ages $65-$ 74 years to more than $30 \%$ in patients ages 85 years or older. ' Most elderly persons also receive additional drugs, which may interfere with other nutrients and food intake and, therefore, thiamin intake.

Owing to the increasing prevalence of heart failure ${ }^{50}$ and the lack of danger of thiamin toxicity, a supplementation trial with thiamin combined with the usual medical therapy in elderly heart failure patients seems to be war- 
ranted. The cost of heart failure is so high $^{51}$ that the addition of a safe and cheap therapeutic option should not be discounted. Such a therapeutic trial could be very advantageous because any favorable effects may be seen within a few days, at minimal cost, and with no side effects.

The prevalence of frank vitamin $B_{1}$ deficiency in cardiac failure patients is not known. A French study ${ }^{52}$ found no significant difference in the thiamin status of 35 elderly hospital inpatients with cardiac failure compared with matched patients with other diagnoses. Nevertheless, $11.5 \%$ of the cardiac failure patients were found to be deficient compared with $6 \%$ in the control group; in addition, thiamin deficiency was more frequent in the functional class of New York Heart Association (NYHA) IV compared with NYHA III.

In clinical practice there is no need to assess the biochemical vitamin $B_{1}$ nutriture in every patient with heart failure because the biochemical assessment of vitamin $B_{1}$ nutriture is difficult and an adequate handling of the blood during the preanalytic stage is rarely assured in a routine hospital setting. The clinical setting (an elderly multimorbid patient on chronic diuretic therapy and inadequate food intake) should be the trigger for the consideration of a thiamin supplementation trial. One central risk indicator for vitamin $B_{1}$ deficiency is poor appetite. The differential diagnosis of a poor appetite is broad; nevertheless, Kwok et al. ${ }^{10}$ identified poor appetite as an indicator of impaired thiamin nutriture in elderly patients with congestive heart failure.

These two new studies ${ }^{12,13}$ will hopefully lead to a revival of interest in this neglected area of medicine. The prevalence of cardiac failure is increasing worldwide and represents a leading cause of morbidity and mortality and impaired quality of life in elderly persons. From the viewpoint of metabolic cardiology, the evidence of the importance of thiamin in cardiac metabolism and function seems convincing. Randomized prospective placebo-controlled studies are still needed. In view of the fast response rate upon supplementation - within a few days - and the increasing "availability" of heart failure patients as subjects, final clarifying evidence can be obtained quickly.

Until there is more convincing evidence, a high level of clinical suspicion and the generous supplementation of thiamin in high-risk patients is probably the best strategy to combat deficiency and lead to an improved quality of life in many patients.

1. van-Kraaij DJ, Jansen RW, de-Gier JJ, et al. Prescription patterns of diuretics in Dutch communitydwelling elderly patients. $\mathrm{Br} \mathrm{J}$ Clin Pharmacol 1998;46:403-7

2. Gilman AG, Rall TW, Nies AS, Taylor P. The pharmacological basis of therapeutics, vol 8. New York: Pergamon Press, 1991;1-1737

3. Brady JA, Rock CL, Horneffer MR. Thiamine status, diuretic medications, and the management of con- gestive heart failure. J Am Diet Assoc 1995;95:5414

4. Seligmann $\mathrm{H}$, Halkin $\mathrm{H}$, Rauchfleisch $\mathrm{S}$, et al. Thiamine deficiency in patients with congestive heart failure receiving long-term furosemide therapy: a pilot study. Am J Med 1991;91:151-5

5. Freye $E$, Hartung $E$. The potential use of thiamine in patients with cardiac insufficiency. Acta Vitaminology Enzymology 1982;4:285-90

6. Bell D, Robertson CE, Muir AL. Carbonated drinks, thiamine deficiency and right ventricular failure. Scott Med J 1987;32:137-8

7. Standl E, Haslbeck $H$, Geser CA, Mehnert $H$. Untersuchungen zur frage einer wechselwirkung von saluretisch wirksamen thiazidderivaten und thiamin bei diabetikern. Klinische Wochenschrift 1968;46:1171-3

8. Kasper H. Der einfluss von diuretika auf die thiaminausscheidung. Bibl Nutr Dieta 1969;11:23-9

9. Leslie D, Gheorghiade $M$. Is there a role for thiamine supplementation in the management of heart failure? Am Heart J 1996;131:1248-50

10. Kwok T, Falconer-Smith JF, Potter JF, Ives DR. Thiamine status of elderly patients with cardiac failure. Age Aging 1992;21:67-71

11. Yui Y, Itokawa Y, Kawai C. Furosemide-induced thiamine deficiency. Cardiovasc Res 1980;14:537-40

12. Rieck J, Halkin $H$, Almog $S$, et al. Urinary loss of thiamine is increased by low doses of furosemide in healthy volunteers. J Lab Clin Med 1999;134: 238-43

13. Lubetsky A, Winaver J, Seligmann $H$, et al. Urinary thiamine excretion in the rat: effects of furosemide, other diuretics, and volume load. J Lab Clin Med 1999;134:232-7

14. Pang JA, Yardumian A, Davies R, Patterson DLH. Shoshin beriberi: an underdiagnosed condition. Intensive Care Med 1986;12:380-2

15. McIntyre N, Stanley NN. Cardiac beriberi: two modes of presentation. BMJ 1971;3:567-9

16. Kawai C, Wakabayashi A, Matsumura T, Yui Y. Reappearance of beriberi heart disease in Japan. Am J Med 1980;69:383-6

17. O'Keeffe ST, Tormey WP, Glasgow R, Lavan JN. Thiamine deficiency in hospitalized elderly patients. Gerontology 1994;40:18-24

18. Akpan T, Peschard S, Brinkane AH, et al. Right heart failure caused by thiamine deficiency (cardiac beriberi). Presse Med 2000;29:240-1

19. Gennery AR, Bartlett K, Hasan A. Thiamine deficiency mimicking acute rejection following cardiac transplantation. Cardiology in the Young 1998;8: 113-5

20. Carney MWP, Barry S. Clinical and subclinical thiamine deficiency in clinical practice. Clinical Neuropharmacol 1984;8:286-93

21. Cappelli V, Bottinelli R, Polla B, Reggiani C. Altered contractile properties of rat cardiac muscle during experimental thiamine deficiency and food deprivation. J Mol Cell Cardiol 1990;22:1095-106

22. Zangen $A$, Shainberg $A$. Thiamine deficiency in cardiac cells in culture. Biochem Pharmacol 1997; 54:575-82

23. Djoenaidi W, Notermans SLH, Dunda G. Beriberi cardiomyopathy. Eur J Clin Nutr 1992;46:227-34 
24. Cook CC, Thomson AD. B-complex vitamins in the prophylaxis and treatment of Wernicke-Korsakoff syndrome. British Journal of Hospital Medicine 1997;57:461-5

25. Butterworth RF, D'Amour M, Bruneau J, et al. Role of thiamine deficiency in the pathogenesis of alcoholic peripheral neuropathy and the WernickeKorsakoff syndrome: an update. In: Palmer TN, ed. Alcoholism: a molecular perspective. New York: Plenum Press, 1991;269-73

26. National Academy of Sciences. Standing Committee on the Scientific Evaluation of Dietary Reference Intakes. Food and Nutrition Board. Dietary reference intakes: thiamin, riboflavin, niacin, vitamin $B_{6}$, folate, vitamin $B_{12}$, pantothenic acid, biotin, and choline. Washington, DC: National Academy Press, 1998;1-425

27. Seitz HK, Suter PM. Ethanol toxicity and the nutritional status. In: Kotsonis FN, Mackey M, Hjelle J, eds. Nutritional toxicology. New York: Raven Press, 1994;95-116

28. Russell RM, Suter PM. Vitamin requirements of the elderly: an update. Am J Clin Nutr 1993;58:4-11

29. Iber FL, Blass JP, Brin M, Leevy CM. Thiamin in the elderly-relation to alcoholism and to neurological degenerative disease. Am J Clin Nutr 1982;36: 1067-82

30. Suter PM, Russell RM. Vitamin requirements of the elderly. Am J Clin Nutr 1987;45:501-12

31. Markkanen T. The effect of acetazolamide and mercaptomerin on the urinary excretion of thiamine, riboflavin and pantothenic acid. Zeitschrift fur Vitamin, Hormon und Fermentforschung 1965;14:726

32. Inara $M$, Ito $T$, Yanagihara $C$, Nishimura $Y$. Wernicke's encephalopathy associated with hemodialysis: report of two cases and review of the literature. Clin Neurol Neurosurg 1999;101:118-21

33. Gibson RS. Principles of nutritional assessment New York: Oxford University Press, 1990;691

34. Shimon I, Almog S, Vered Z, et al. Improved left ventricular function after thiamine supplementation in patients with congestive heart failure receiving long-term furosemide therapy. Am J Med 1995;98: 485-90

35. Levy WC, Soine LA, Huth MM, Fishbein DP. Thiamine deficiency in congestive heart failure. Am J Med 1992;93:705-6

36. Yue QY, Beermann B, Lindstrom B, Dyquist O. No difference in blood thiamine diphosphate levels between Swedish Caucasian patients with cardiac failure treated with furosemide and patients without heart failure. J Intern Med 1997;242:491-5

37. Morrow LE, Grimsley EW. Long-term diuretic therapy in hypertensive patients: effects on serum homocysteine, vitamin $B_{6}$, vitamin $B_{12}$ and red blood cell folate concentrations. South Med J 1999;92: 866-70

38. Suter PM, Haller J, Hany A, Vetter W. Diuretic use: a risk factor for subclinical thiamine deficiency in elderly patients. The Journal of Nutrition, Health \& Aging 2000;4:69-71

39. Gubler JC. Thiamin. In: Machlin LJ, ed. Handbook of vitamins: nutritional, biochemical, and clinical aspects. New York: Marcel Dekker Inc, 1992;24597

40. Wodak A, Richmond R, Wilson A. Thiamin fortification and alcohol. Med J Aust 1990;152:97-9

41. Stefikova K, Spustova V, Dzurik R. Acute effect of hydrochlorothiazide on renal calcium and magnesium handling in postmenopausal women. Physiol Res 1999;48:327-30

42. Crippa G, Sverzellati E, Giorgi-Pierfranceschi M, Carrara GC. Magnesium and cardiovascular drugs: interactions and therapeutic role. Ann Ital Med Int 1999;14:40-5

43. Quamme GA. Renal magnesium handling: new insights in understanding old problems. Kidney Int 1997;52:1180-95

44. Altura BM, Altura BT. Magnesium and cardiovascular biology: an important link between cardiovascular risk factors and atherogenesis. Cell Mol Biol Res 1995;41:347-59

45. Sueta CA, Patterson JH, Adams KF. Antiarrhythmic action of pharmacological administration of magnesium in heart failure: a critical review of new data. Magnes Res 1995;8:389-401

46. Dyckner T, Ek B, Nyhlin H, Wester PO. Aggravation of thiamine deficiency by magnesium depletion. Acta Med Scand 1985;218:129-31

47. McLean J, Manchip S. Wernicke's encephalopathy induced by magnesium depletion. Lancet 1999; 353:1768

48. Durlach J, Bac P, Durlach V, et al. Magnesium status and aging: an update. Magnes Res 1998;11:2542

49. Zangen A, Botzer D, Zangen R, Shainberg A. Furosemide and digoxin inhibit thiamine uptake in cardiac cells. Eur J Pharmacol 1998;361:151-5

50. Goff DC, Pandey DK, Chan FA, et al. Congestive heart failure in the United States: is there more than meets the ICD code? The Corpus Christi Heart Project. Arch Intern Med 2000;160:197-202

51. O'Connell JB. The economic burden of heart failure. Clin Cardiol 2000;23(suppl 3):6-10

52. Pfitzenmeyer P, Guilland JC, d'Athis P, et al. Thiamine status of elderly patients with cardiac failure including the effects of supplementation. Int $\mathrm{J} \mathrm{Vi-}$ tam Nutr Res 1994;64:113-8 